\title{
Dual-Band Beams Generation with Metasurface based on the EFIE
}

\author{
Modeste Bodehou ${ }^{1}$, David González-Ovejero ${ }^{2}$, Christophe Craeye ${ }^{1}$ and Isabelle Huynen ${ }^{1}$ \\ ${ }^{1}$ Université catholique de Louvain: ICTEAM Institute, Place du Levant, 3, 1348, Louvain-la-Neuve, Belgium \\ ${ }^{2}$ Univ Rennes, CNRS, IETR (Institut d'Électronique et de Télécommunications de Rennes), \\ UMR 6164, 35000 Rennes, France \\ ${ }^{1}$ modeste.bodehou, christophe.craeye, isabelle.huynen@uclouvain.be, ${ }^{2}$ david.gonzalez-ovejero@univ-rennes1.fr
}

\begin{abstract}
This paper investigates the generation of dual-band beams by modulated metasurface antennas. The developed technique combines a semi-separable model dependence of the sheet impedance (with respect to frequency and patch parameters) with the resolution of the electric field integral equation (EFIE). This allows us to express the impedance modulation at one frequency as a function of the modulation at the second frequency. Then, we can formulate the EFIE at both frequencies while referring to the surface impedance at a single frequency, still fully taking into account the frequency dispersion of the substrate. The method is illustrated through the synthesis of a circularly polarized conical beam and a broadside pencil beam at two different frequencies. Numerical validation with two different Method of Moments (MoM) codes is provided.
\end{abstract}

\section{INTRODUCTION}

Surface waves (SW) to leaky waves (LW) transformation using metasurfaces (MTS) has been the subject of intense research over the past decade [1]-[4]. This interest is due to the multiple advantages offered by this type of antennas (low profile, low cost, limited power losses, etc). A lot of progress has been made in the synthesis of modulated MTS antennas, which include the generation of high-gain polarized beams [4], shaped beam synthesis [5]-[8], multibeam with multiple feeds [9], [10] etc.

However, MTS antennas are relatively narrowband given the highly frequency dispersion of the SW. This means that a change in the frequency of operation strongly impacts the SW mode supported by the MTS. The interaction between the modulation parameters, and the new SW mode is no longer consistent with the desired radiation effect. In many contexts, multiple-band operation is required since it allows one to multiplex several channels at different frequencies, while sharing the same MTS aperture. In [11], the authors use holographic theory to design a dual-band antenna based on a scalar (isotropic) MTS. In particular, the method consists of superimposing several holograms corresponding to different beams and different feed positions. However, scalar MTSs are limited in terms of beam polarization control as proven in [4].

Reference [12] superimposes tensorial (anisotropic) impedances associated with broadside pencil beams and different feed positions. However, the case of beams with different shapes is not treated
The method proposed here is based on a direct solution of the electric field integral equation (EFIE) at the frequencies of interest and can be used for one or multiple feeds. This leads to an over-constrainted system of equations. The design of a MTS radiating a conical beam and a broadside pencil beam at different frequencies is presented. A general discussion and possible improvements on the method is also provided.

The paper is structured as follows. Section II briefly recalls the EFIE based MTS synthesis at one frequency. Section III extends the EFIE synthesis method to multiple frequencies. Section IV presents some numerical results and section $\mathrm{V}$ concludes the paper.

\section{SINGLE BAND OPERATION}

Let us consider a circular MTS antenna modeled as a sheet transition impedance boundary condition laying on a grounded substrate. The usual polar system of coordinates is used to identify each point on the MTS. The current distribution $\mathbf{J}$ and the aperture electric field is linked to the sheet impedance $\underline{\underline{\mathbf{Z}}}_{S}$ on the MTS plane through the following EFIE [13], [14]:

$$
\hat{\mathbf{n}} \times\left[\iint_{S^{\prime}} \underline{\underline{\mathbf{G}}}^{E J}\left(\boldsymbol{\rho}, \boldsymbol{\rho}^{\prime}\right) \mathbf{J}\left(\boldsymbol{\rho}^{\prime}\right) d S^{\prime}-\underline{\underline{\mathbf{Z}}}_{S}(\boldsymbol{\rho}) \mathbf{J}(\boldsymbol{\rho})\right]=-\hat{\mathbf{n}} \times \mathbf{E}_{i}
$$

where $\underline{\mathbf{G}}^{E J}$ is the dyadic Green's function of the grounded substrate, $\mathbf{E}_{i}$ is the MTS excitation and $\hat{\mathbf{n}}$ is the normal to the surface. The polar unit vector is used for the current distribution and surface impedance modulation expansion [14]. Fourier-Bessel basis functions (FBBFs) are a class of scalar functions defined on the whole circular domain [14]. Each component of the current distribution and the surface impedance modulation are now discretized into FBBFs as detailed in equations (21)-(23) in [7]. The synthesis problem consists in, first, computing the objective surface current distribution based on the desired radiation pattern. Next, one must solve the EFIE to derive the corresponding surface impedance, while constraining the surface impedance to be anti-Hermitian so as to avoid losses on the sheet. This leads to a system of equations written in compact form as:

$$
\underline{\underline{\mathbf{Z}}}^{S} \underline{\mathbf{X}}_{S}=\underline{\mathbf{U}}
$$


where the vector $\underline{\mathbf{X}}_{S}$ is the unknown sheet impedance discretized into FBBFs. The matrix $\underline{\mathbf{Z}}^{S}$ and the vector $\underline{\mathbf{U}}$ depend on the radiation pattern, the excitation, and on the average impedance. The reader is referred to [7] for the formulation details.

\section{DuAl BAND OPERATION}

To extend this formulation to dual-band operation, one needs to model the frequency variation of the sheet impedance with respect to the used patches. Let us denote by $\mathbf{p}$ a vector characterizing the patch parameters used to implement the surface impedance. In turn, $Z_{S}(\mathbf{p}, \omega)$ denotes each component of the sheet impedance corresponding to a given patch $\mathbf{p}$ at a given radian frequency $\omega$. For an elliptical patch for example, p represents the semi-major axis, the semi-minor axis and the orientation of the ellipse. The frequency variation of each component of the sheet impedance tensor is now characterized by the following model [15]:

$$
Z_{S}(\mathbf{p}, \omega)=a(\omega) Z_{S}(\mathbf{p})+b(\omega)
$$

where $a(\omega)$ and $b(\omega)$ are arbitrary non linear functions. This model is very accurate provided that we stay in the homogenizable region [15], i.e, considering the unit cell very small with respect to the wavelength of operation. Equation (3) allows us to express the modulation parameters at a given frequency $\omega_{2}$ with respect to the modulation at a reference frequency $\omega_{1}$. One can therefore proceed to the synthesis by stacking the system of equations corresponding to each frequency. In these stacked systems, we obtain the modulations at other frequencies with respect to the one at a given reference frequency using (3). The matrix corresponding to the obtained system of equations can then be written as [15]:

$$
\underline{\underline{\mathbf{Z}}}^{S}=\left[\underline{\underline{\mathbf{Z}}}^{S}\left(\omega_{1}, V_{1}, \mathbf{F}_{1}\right) ; \underline{\underline{\mathbf{Z}}}^{S}\left(\omega_{2}, V_{2}, \mathbf{F}_{2}\right)\right]
$$

and $\underline{\mathbf{U}}$ takes the form:

$$
\underline{\mathbf{U}}=\left[\underline{\mathbf{U}}\left(\omega_{1}, V_{1}, \mathbf{F}_{1}\right) ; \underline{\mathbf{U}}\left(\omega_{2}, V_{2}, \mathbf{F}_{2}\right)\right]
$$

where $\mathbf{F}_{i}$ and $V_{i}$ are respectively the radiation pattern and the excitation used at the radian frequency $\omega_{i}$. The obtained system of equations is then solved in a least-squares sense. Note that, if one is interested in stronger requirements for a given radiation pattern in comparison with the others, one can perform a weighted least-squares.

\section{NumeriCAl Results}

In this section, we present a MTS design capable of radiating two different beams at two different frequencies. The first one is a right-handed circularly polarized (RHCP) conical beam (pointing at $\theta=20^{\circ}$ from broadside), and should be generated at $f_{1}=24 \mathrm{GHz}$. The second beam is a RHCP pencil beam generated at $f_{2}=20 \mathrm{GHz}$. The MTS is printed on a substrate with relative permittivity $\epsilon_{r}=3.66$ and thickness $1.524 \mathrm{~mm}$. The antenna radius is $10 \lambda_{1}$, where $\lambda_{1}$ is the free-space wavelength at $24 \mathrm{GHz}$. At both frequencies, the antenna is excited from the center with an infinitesimal vertical dipole placed in the middle of the substrate [14], [13].
This excitation model is sufficient to provide an excellent idea about the radiation pattern obtained with practical feeds, as already demonstrated in several papers [4], [16]. The reactance is modulated around an average value $X_{0}=-1301 \Omega$ at $24 \mathrm{GHz}$ and we used $a^{\rho \rho}\left(w_{2}\right)=1.2, a^{\rho \phi}\left(w_{2}\right)=1.1$, $b^{\rho \rho}\left(w_{2}\right)=b^{\rho \phi}\left(w_{2}\right)=0$.

Fig. 1 shows the designed reactance modulation at $24 \mathrm{GHz}$. Fig. 2 illustrates the simulated radiation pattern obtained with

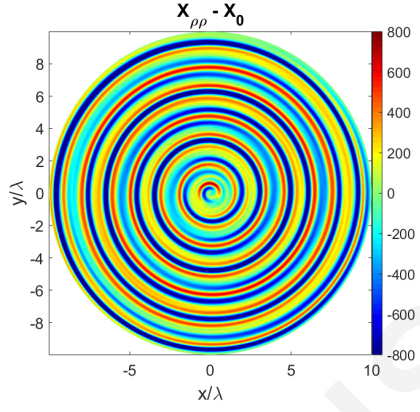

(a) $\rho \rho$ modulation

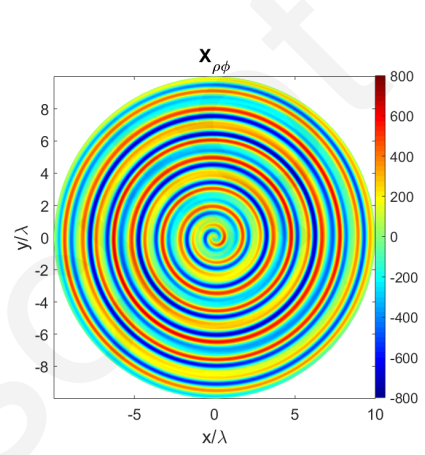

(b) $\rho \phi$ modulation
Fig. 1: Reactance modulation at $24 \mathrm{GHz}$.

two independent MoM codes at $f_{1}=24 \mathrm{GHz}$. The first code is based on Gaussian ring basis functions (GRBF) [13] and the second one uses Fourier-Bessel basis functions (FBBF) [14]. The latter functions consist in Gaussian-type rings and linear azimuthal phase. Their spectrum can be written in analytical form. Hence, they enable a closed-form evaluation of the MoM impedance matrix's entries and lead to an efficient solution of the MoM system of equations. One can observe a good agreement between the two simulation results except the side lobes level. The difference is due to the evolution of the currents at a distance very close to the feed. Indeed GRBFs allow a more accurate current evaluation close to the feed because of the possible zero admissible current (see [14] for a detailed discussion regarding this aspect). The obtained aperture efficiency is about $79 \%$. This efficiency is computed in comparison with the power density radiated by an ideal conical beam antenna of the same radius.

Fig. 3 shows the radiation pattern at $20 \mathrm{GHz}$. The directivity obtained with GRBF code is $2 \mathrm{~dB}$ higher than that of FBBF code due to the better treatment of GRBFs for the currents near the center, as previously mentioned.

\section{A. Improvement of the method}

One of the main advantages of the proposed EFIE approach is the fact that the method is systematic and starts directly from the radiation pattern, not from the aperture electric field. That means, we specified at input the desired radiation pattern in amplitude, phase and polarization at the frequencies of interest. Despite this flexibility is useful for near-field applications (see some near-field designs in [17]), the phase distribution is usually less or even not important for antenna (far-field) applications. Here, we specified for the conical beam, a linear phase evolution along the azimuthal direction. The simulated 


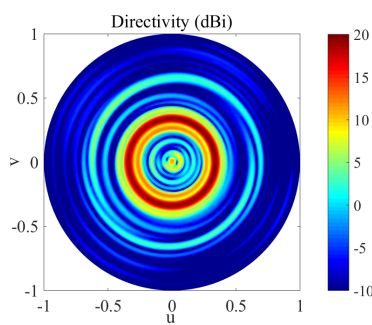

(a) Directivity GRBF

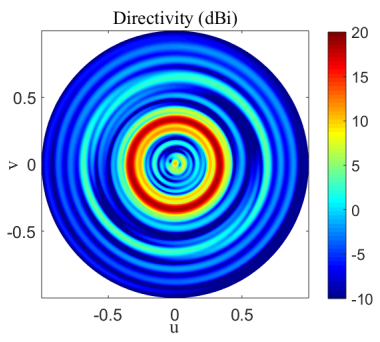

(b) Directivity FBBF

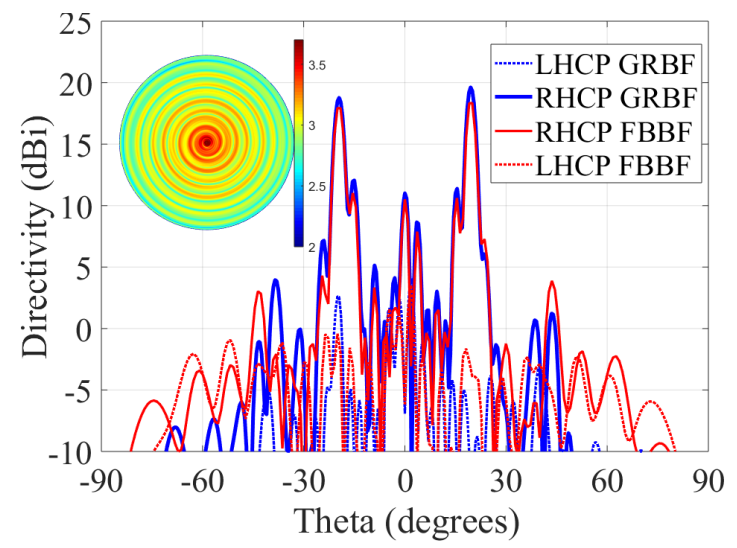

(c) Directivity cut in the plane $\phi=0$. The inset disk represents the absolute value of the current distribution on the MTS in logarithm scale.

Fig. 2: Radiation pattern at $24 \mathrm{GHz}$.

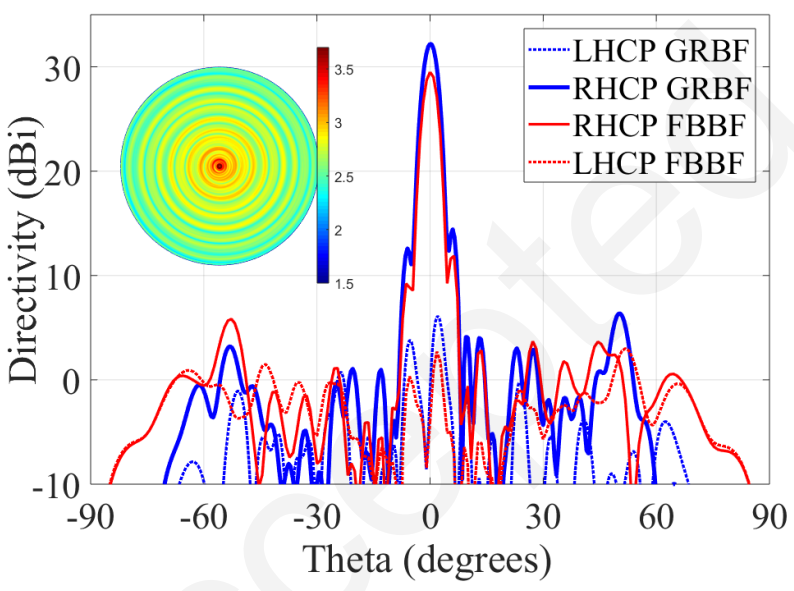

Fig. 3: Radiation pattern at $20 \mathrm{GHz}$. The inset disk represents the absolute value of the current distribution on the MTS in logarithm scale.

pattern phase evolution of the designed MTS is represented in Fig. 4. One can see an excellent agreement with respect to the prescribed phase. It is worth mentioning that the designed pattern amplitude and polarization depends on the prescribed phase. When one is interested in realizing complex radiation patterns at multiple frequencies, with strong requirements, the prescribed phase evolution should be optimized to get better results. The phase optimization problem is a complex issue that deserves further research.

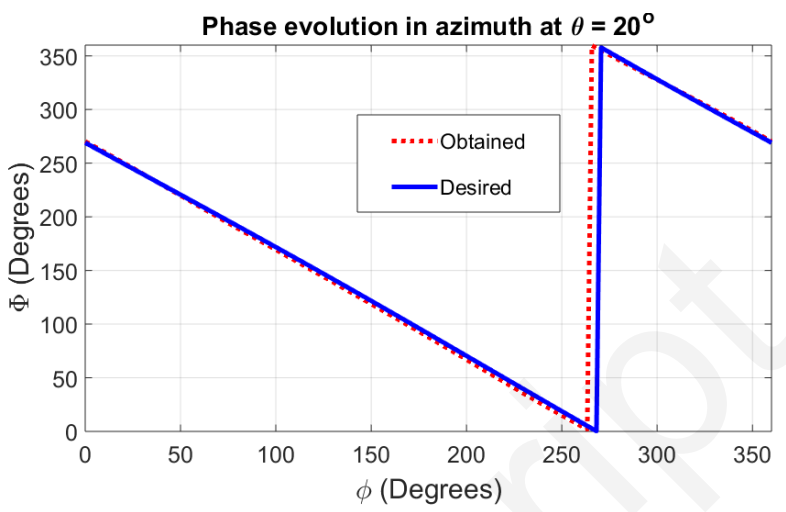

Fig. 4: Conical beam radiation pattern phase evolution at 24 $\mathrm{GHz}$ and $\theta=20^{\circ}$

\section{CONCLUSION}

We have presented an algorithm to generate shaped beams (in amplitude, phase and polarization) at multiple frequencies with modulated metasurface antennas. The method relies on the resolution of the EFIE and fully takes into account the metasurface frequency dispersion. Such metasurfaces can be used to satisfy the requirements of different applications at different frequencies, while employing the same aperture. A very good agreement has been obtained with an independently developed MoM software, making use of Gaussian Ring basis functions. The latter better deals with the current very close to the feed point in comparison with Fourier-Bessel basis functions.

\section{ACKNOWLEDGMENT}

This research has been supported by a FRIA grant from the Belgium FNRS fund.

\section{REFERENCES}

[1] B. H. Fong, J. S. Colbrun, J. J. Ottusch, J. L. Visher and D. F. Sievenpiper "Scalar and tensor holographic artificial impedance surfaces," IEEE Trans. Antennas Propag., vol. 58, no. 10, pp. 3212-3221, Oct. 2010.

[2] A. M. Patel, A. Grbic, "A printed leaky-wave antenna based on a sinusoidally-modulated reactance surface", IEEE Trans. Antennas Propag., vol. 59, no. 6, pp. 2087-2096, Jun. 2011.

[3] S. Maci, G. Minatti, M. Casaletti and M. Bosiljevac, "Metasurfing: Addressing waves on impenetrable metasurfaces," IEEE Antennas Wireless Propag. Lett., vol.10, pp.1499-1502, Feb. 2012.

[4] G. Minatti, M. Faenzi, E. Martini, F. Caminita, P. De Vita, D. GonzálezOvejero, M. Sabbadini and S. Maci, "Modulated metasurface antennas for space: synthesis, analysis and realizations," IEEE Trans. Antennas Propag., vol. 63, no. 4, pp. 1288-1300, April 2015.

[5] G. Minatti, F. Caminita, E. Martini, M. Sabbadini and S. Maci, "Synthesis of modulated-metasurface antennas with amplitude, phase, and polarization control," IEEE Trans. Antennas Propag., vol. 64, no. 9 pp. 39073919, Sep. 2016.

[6] M. Teniou, H. Roussel, N. Capet, G.-P. Piau, and M. Casaletti, "Implementation of radiating aperture field distribution using tensorial metasurfaces," IEEE Trans. Antennas Propag., vol. 65, pp. 5895-5907, Sep. 2017 
[7] M. Bodehou, C. Craeye, E. Martini, and I. Huynen, "A Quasi-direct method for the surface impedance design of modulated metasurface antennas," IEEE Trans. Antennas Propag., vol. 67, no. 1, pp. 24-36, Jan. 2019.

[8] M. Bodehou, C. Craeye, and I. Huynen, "Electric field integral equationbased synthesis of elliptical-domain metasurface Antennas," IEEE Trans. Antennas Propag., vol. 67, no. 2, pp. 1270-1274, Feb. 2019.

[9] D. González-Ovejero, G. Minatti, G. Chattopadhyay and S. Maci, "Multibeam by metasurface antennas," IEEE Trans. Antennas Propag., vol. 65, no. 6, pp. 2923-2930, June 2017.

[10] M. Bodehou, E. Martini, S. Maci, I. Huynen, and C. Craeye "Multibeam and beam scanning with modulated metasurfaces," Accepted for publication in IEEE Trans. Antennas Propag., Aug 2019.

[11] Y. Li, A. Li, T. Cui, and D. Sievenpiper, "Multi-wavelength multiplexing hologram designed using impedance metasurfaces," IEEE Trans. Antennas Propag., vol. 66, no. 11, pp. 6408-6413, Nov. 2018.

[12] M. Faenzi, D. González-Ovejero, F. Caminita, and S. Maci "Dual-band self-diplexed modulated metasurface antennas," Proc. EUCAP, Apr. 2018.

[13] D. González-Ovejero and S. Maci, "Gaussian ring basis functions for the analysis of modulated metasurface antennas," IEEE Trans. Antennas Propag., vol. 63, pp. 3982-3993, Sept. 2015.

[14] M. Bodehou, D. González-Ovejero, C. Craeye and I. Huynen, "Method of Moments simulation of modulated metasurface antennas with a set of orthogonal entire-domain basis functions," IEEE Trans. Antennas Propag., vol. 67, no. 2, pp. 1119-1130, Feb. 2019.

[15] M. Bodehou, C. Craeye, and I. Huynen, "Multi-frequency band synthesis of modulated metasurface antennas," submitted to IEEE Antennas Wireless Propag. Lett., Jul. 2019.

[16] M. Bodehou, C. Craeye, H. Bui-Van, and I. Huynen, "Fourier-Bessel basis functions for the analysis of elliptical domain metasurface antennas," IEEE Antennas Wireless Propag. Lett., vol. 17, pp. 675-678, April. 2018.

[17] M. Bodehou, C. Craeye, and I. Huynen, "Near-field shaping by leakywave metasurfaces: OAM and Bessel beams synthesis," Proc. ICEAA, Oct. 2019. 